\title{
Electrospun Aspirin/Eudragit/Lipid Hybrid Nanofibers for Colon-targeted Delivery Using an Energy-saving Process
}

\author{
WANG Yibin ${ }^{1 \#}$, TIAN Liang $^{1 \#}$, ZHU Tianhao ${ }^{1}$, MEI Jing ${ }^{1}$, \\ CHEN Zezhong ${ }^{1 \bowtie}$ and YU Deng-Guang ${ }^{1,2 \bowtie}$
}

\author{
Received January 4, 2021 \\ Accepted March 20, 2021 \\ (c) Jilin University, The Editorial Depart- \\ ment of Chemical Research in Chinese \\ Universities and Springer-Verlag $\mathrm{GmbH}$
}

B oth electrospinning apparatus and their commercial products are extending their applications in a wide variety of fields. However, very limited reports can be found about how to implement an energy-saving process and in turn to reduce the production cost. In this paper, a brand-new type of coaxial spinneret with a solid core and its electrospinning methods are developed. A novel sort of medicated Eudragit/lipid hybrid nanofibers are generated for providing a colon-targeted sustained release of aspirin. A series of characterizations demonstrates that the as-prepared hybrid nanofibers have a fine linear morphology with the aspirin/lipid separated from the matrix Eudragit to form many tiny islands. In vitro dissolution tests exhibit that the hybrid nanofibers are able to effectively prevent the release of aspirin under an acid condition $(8.7 \% \pm 3.4 \%$ for the first two hours), whereas prolong the drug release time period under a neutral condition $(99.7 \pm 4.2 \%$ at the seventh hour). The energy-saving mechanism is discussed in detail. The prepared aspirin-loaded hybrid nanofibers can be further transferred into an oral dosage form for potential application in countering COVID-19 in the future.

Keywords Coaxial spinneret; Polymer/lipid hybrid fibers; Colon-targeted; Aspirin; COVID-19

\section{Introduction}

Electrospinning, an abbreviation of electrostatic spinning, belongs to an electrohydrodynamic atomization(EHDA) $\operatorname{method}^{[1-3]}$. Based on the strong, intensive and fast interactions between the electrostatic energy and the electrospinnable polymeric fluid, solid nanofibers can be generated within the time scale of several milliseconds ${ }^{[4-9]}$. This kind of "top-down" nano fabrication method has its unique advantages, such as a single-step and straightforward preparation process, convenient for functionalization, after treatment, and further conversion ${ }^{[10-14]}$. Thus, it is no strange that it has rapidly penetrated into almost all the applied scientific fields with the surge of nano science and technology

\footnotetext{
$\triangle$ YU Deng-Guang

ydg017@usst.edu.cn

$\triangle$ CHEN Zezhong

zzhchen@usst.edu.cn

\# These authors contributed equally to this work.

1. School of Materials Science \& Engineering, University of Shanghai for

Science \& Technology, Shanghai 200093, P. R. China;

2. Shanghai Engineering Technology Research Center for High-Performance Medical Device Materials, Shanghai 200093, P. R. China
}

during the past three decades ${ }^{[15,16]}$. Presently, more and more attention has been paid to the creation of nanofibers on a large scale and their potential commercial products ${ }^{[4-9]}$. Under this situation, energy saving for reducing the cost should be a very important issue, particularly, electrospinning treats and converts materials by direct application of the high-voltage electrostatic energy.

The application of drug-loaded electrospinning nanofibers for controlling drug delivery dates back to 2002, when Kenawy et al.[17] reported the sustained release effect of drug-loaded nanofibers. Afterwards, with the development of electrospinning technology, a variety of characteristic drugcontrolled release profiles were studied and reported, for example, time-controlled or rate-controlled release including rapid release, delayed-release, long-term degradation release, multi-phase controlled release, and location-controlled release including colon targeting and local positioning ${ }^{[18-21]}$. Once electrospinning methods based on fluid properties, such as solution electrospinning, melt electrospinning, emulsion electrospinning and suspension electrospinning, and electrospinning methods based on the simultaneous combination of several fluids, such as coaxial electrospinning, side-by-side electrospinning, tri-axial electrospinning, tri-fluid side-by-side electrospinning and other multiple-fluid processes appeared, they were swiftly applied for potential applications in the field of drug controlled release ${ }^{[22-26]}$.

According to the backgrounds mentioned above, a new type of coaxial spinneret with a solid core and its electrospinning methods were developed in this investigation. A novel sort of hybrid nanofibers were generated for simultaneously controlling the drug release location and the drug release rate. Due to the wide application of aspirin in the treatment of COVID-19 patients, as well as the fact that the side effects of aspirin came from two aspects(strong stimulation to the stomach and toxic side effects caused by excessive dose $)^{[27-32]}$, it was chosen as the model drug here. Moreover, Eudragit S100, an enteric soluble polymer, was selected as the filament-forming polymeric matrix proposed to prevent the release of aspirin in the stomach. What's more, hydrophobic lecithin was added to the spinnable solution to improve the sustained-release performances of aspirin from the created 


\section{Experimental}

\subsection{Materials and Methods}

Asprin(purity $=99 \%$, lot number: 2017087 ) was purchased from Shanghai Aladdin Bio-Chem Technology Co., Ltd.(Shanghai, China). Lecithin(medicinal egg yolk extract) was bought from Sinopharm Chemical Reagent Co., Ltd.(Shanghai, China). Eudragit ${ }^{\circledR}$ S100 was obtained from Röhm Chemical Co., Ltd.(Shanghai, China). N,N-Dimethylacetamide, absolute ethanol and other chemical agents were bought from Sinopharm Chemical Reagent Co., Ltd.(Shanghai, China). All chemicals were of analytical grade, and water was doubly distilled before use.

\subsection{Preparation of Hybrid Nanofibers}

The homemade electrospinning system was composed of four parts. A high-voltage $(60 \mathrm{kV} / 2 \mathrm{~mA})$ power supply(ZGF2000, Shanghai Sute Corp., Shanghai, China) and a syringe pump(KDS100, Cole-Parmer, IL, USA) were used. The spinneret was homemade. A piece of aluminum foil was wrapped up on a cardboard used as the fiber collector.

Preparation of spinnable solution: the concentrations of aspirin, lecithin and Eudragit ${ }^{\circledR}$ S100 dissolved in the mixed solvent were 3.0, 3.0 and $12.0 \mathrm{~g} / \mathrm{mL}$, respectively. Based on the pre-experiments, the processing parameters of electrospinning were fixed as follows: the applied voltage was set at $14 \mathrm{kV}$; the flow rate was adjusted at $1.0 \mathrm{~mL} / \mathrm{h}$; and the distance between the spinneret and the collector was fixed at $20 \mathrm{~cm}$.

\subsection{Characterization of Hybrid Nanofibers}

The morphology and structure of fibers: the surface morphology of fibers was observed on a scanning electron microscope(SEM, Quanter 450, FEI, USA). The sample was sprayed with gold for $60 \mathrm{~s}$. The diameters of fibers were statistically analyzed by the Image software(National Institutes of Health, USA). The internal structure of fibers was observed on a transmission electron microscope(TEM, JEM 2100F, JEOL, Tokyo, Japan).

The components' physical state and their compatibility in the fibers: the physical states of the components in the raw materials and fibers were detected on an X-ray diffractometer(XRD, Bruker X-ray diffractometer, Karlsruhe, Germany). All samples were measured within a $2 \theta$ angle range of $5-60^{\circ}$. The compatibility of components in fibers was detected on a Perkin-Elmer FTIR Spectrometer(Spectrum 100 FTIR spectrometer, PerkinElmer, Billerica, USA) over the range

\subsection{Functional Application of Hybrid Nanofibers}

In vitro dissolution tests measuring drug-controlled release profiles from the hybrid nanofibers were carried out by paddle method according to Method II, as described in Chinese Pharmacopoeia(2015 Ed.). The fiber samples were weighed for $0.2 \mathrm{~g}$ and firstly placed in an $\mathrm{HCl}$ solution $(\mathrm{pH}=2$, simulated artificial gastric fluid without enzyme) for $2 \mathrm{~h}$, then transferred into the phosphate buffer saline(PBS, $\mathrm{pH}=7.2,0.1$ $\mathrm{mol} / \mathrm{L}$, enzyme-free artificial intestinal fluid). The temperature of the in vitro dissolution apparatus(ZRS-8G, Radio Factory of Tianjin University) was set at $(37.0 \pm 0.5){ }^{\circ} \mathrm{C}$ and the paddle rotation speed was maintained at $(50 \pm 2) \mathrm{r} / \mathrm{min}$. At the predetermined time point, $5.0 \mathrm{~mL}$ of samples were withdrawn and the same-volume PBS medium was replenished. The samples were filtered through a $0.22 \mu$ m cellulose membrane(Millipore, USA). After direct and appropriate dilution to the filtrate, samples were analyzed at $\lambda_{\max }=278 \mathrm{~nm}$ using a UV-Vis spectrophotometer(UV-2102PC, Unico Instrument Co., Ltd., Shanghai, China). The drug concentration and cumulative release percentage were calculated according to a calibration curve. All experiments were repeated 6 times.

\section{Results and Discussion}

\subsection{Preparation of New-type Spinneret}

The homemade coaxial spinneret with a solid core is shown in Fig.1. The arrangements of inner capillaries of the spinneret are diagrammed in Fig.1(A). A full digital image of the spinneret and the exit of its nozzle are exhibited in Fig.1(B) and Fig.1(C), respectively. The spinneret was comprised of two metal capillaries, the epoxy resin adhesive and a Teflon solid rod. The two metal tubes were $19 \mathrm{G}$ and $25 \mathrm{G}$ stainless steel capillary tubes. The 25G internal capillary was inserted
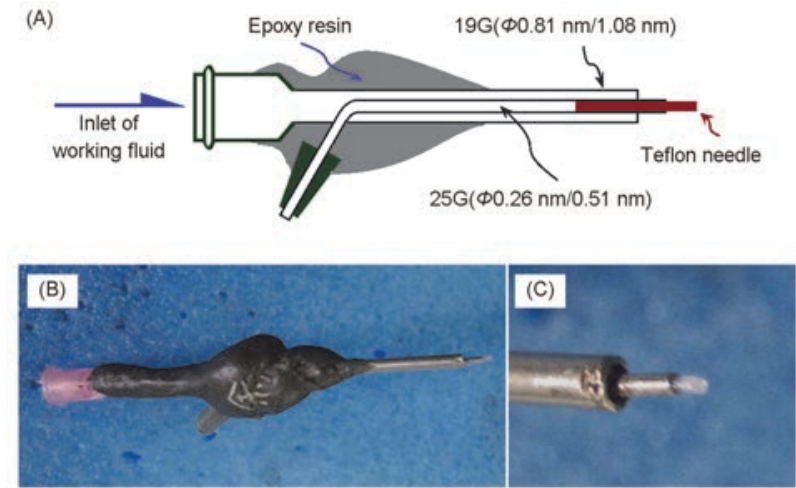

Fig.1 Coaxial spinneret with a solid Teflon core rod

(A) The arrangements of inner capillaries; (B) a full digital image of the spinneret; (C) a digital image about the nozzle of the spinneret. 
into the 19G external capillary along the tube wall. The external tube inlet slot and the internal tube inlet bolt were insulated rubber. The $0.26 \mathrm{~mm}$ Teflon solid rod was inserted by the $25 \mathrm{G}$ internal tube, forming the 'free surface' of fluid diversion. Despite a small part of $19 \mathrm{G}$ external metal tube that was kept outside, all other positions contacted with the environment were insulated polymers. The working fluid was pushed through the syringe from the slot connected with the 19G external sheath tube.

\subsection{Implementation of Electrospinning}

In an electrospinning system or a set of electrospinning equipment, the spinneret is the most important component ${ }^{[33]}$. The other three devices, the high voltage power supply, the syringe pump and the collector, respectively, operate for providing high-voltage electrostatic energy, measuring and pushing the working fluid accurately, and receiving the prepared nanofibers. The structure of the spinneret determines the distinction and classification of the electrospinning process, for example, the spinneret with a core-sheath structure refers to coaxial electrospinning, the spinneret with a Janus structure refers to side-by-side electrospinning, the spinneret with a complicated multi-compartment structure refers to multi-fluid electrospinning, and the "free surface" of the fluid refers to needle-less electrospinning. The spinneret in this study integrated the advantages of "free surface" into needle electrospinning. Based on this spinneret, the homemade highvoltage electrospinning system is shown in Fig.2(A). Fig.2(B) shows how the spinneret is connected with the high voltage electrostatic power supply. The working fluid was directly connected and pushed through the syringe pump, while the spinneret was immobilized by the syringe, which prevented unsafe accidents like short circuit resulted from slipping. High voltage electrostatic energy could be directly introduced by an alligator clip. When the high voltage was not applied, droplets with long axes in a vertical direction were formed due to their own gravity and the adhesion between them and the solid surface of the spinneret[inset in Fig.2(C)]. While the applied

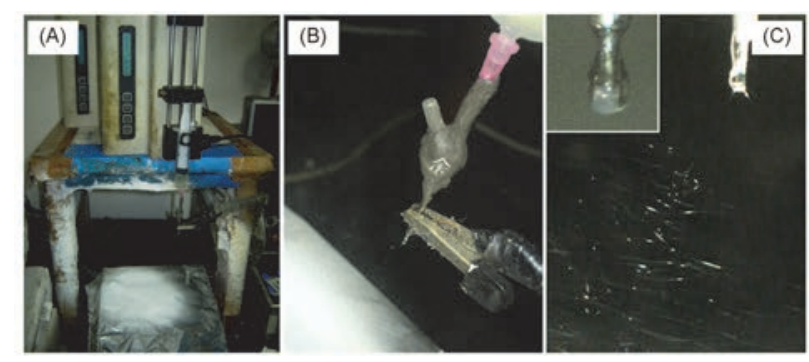

Fig.2 Implementation of the electrospinning

(A) The homemade electrospinning system; (B) the connection of spinneret with high voltage and working fluid; (C) a typical image of the working process(inset shows a liquid droplet without the applied voltage)(magnifications $\times 12$ ). voltage was $8.2 \mathrm{kV}$, a typical and stable nanofiber preparation process could be observed. As shown in Fig.2(C), a typical working fluid trajectory is clear, which was captured by a digital camera at 12-times magnification. This process was similar to that of traditional needle electrospinning, in which three classical procedures were carried on completing the fast material conversion from liquid fluid to solid polymer fiber, namely Taylor cone, straight fluid and instable region of highfrequency stretching and bending.

\subsection{Morphology and Structure of the Prepared Nanofibers}

The prepared aspirin/Eudragit/lecithin hybrid nanofibers were observed through SEM. The images taken at a magnification of 2000 and 40000 are shown in Fig.3(A) and (B), respectively. As shown in Fig.3(A), the prepared hybrid nanofibers had good linear morphology. No spindle or beadson-a-string phenomenon was found. Through analyzing the image, the statistical diameter of fibers was $(760 \pm 130)$ $\mathrm{nm}[$ Fig.3(C)]. The diameter distribution of fibers was narrow and uniform. While observing the nanofibers at high magnification in SEM, some inconspicuous "gray spots" were found distributed on the surface, which should be the tiny lipid particles produced by the solid phase separation of lecithin and aspirin during the electrospinning process. Therefore, the nanofibers were further observed at a 100000 magnification through TEM, whose pattern is shown in Fig.3(D). Numerous tiny white spots were densely dispersed in the fiber, which further proved the phenomenon of solidphase separation.

Electrospinning is essentially a physical process, in which the working fluid is subjected to the high-voltage electrostatic field force, leading to instantaneous drying ${ }^{[34]}$. As the drying process is extremely fast, in general, the dispersion state of different solutes at the molecular level in the working fluid can be effectively "transferred" into the solid nanofibers. Therefore, electrospinning is widely used in the preparation and application of insoluble drug solid dispersion ${ }^{[35,36]}$. However, due to the effects of compatibility, solute concentration and other factors between different solute molecules, the phenomenon of solid-phase separation between additives and fiber substrates occurs sometimes during the instantaneous solidification of the electrospinning process or the storage process of fibers. The TEM samples in this study were prepared and analyzed immediately after preparation, so the gray particles were supposed to be produced in the working process. It was also due to the existence of phase separation particles that the prepared multi-component nanofibers were not the traditional homogeneous nanofibers, but the multi-component and multi-phase hybrid nanofibers. 

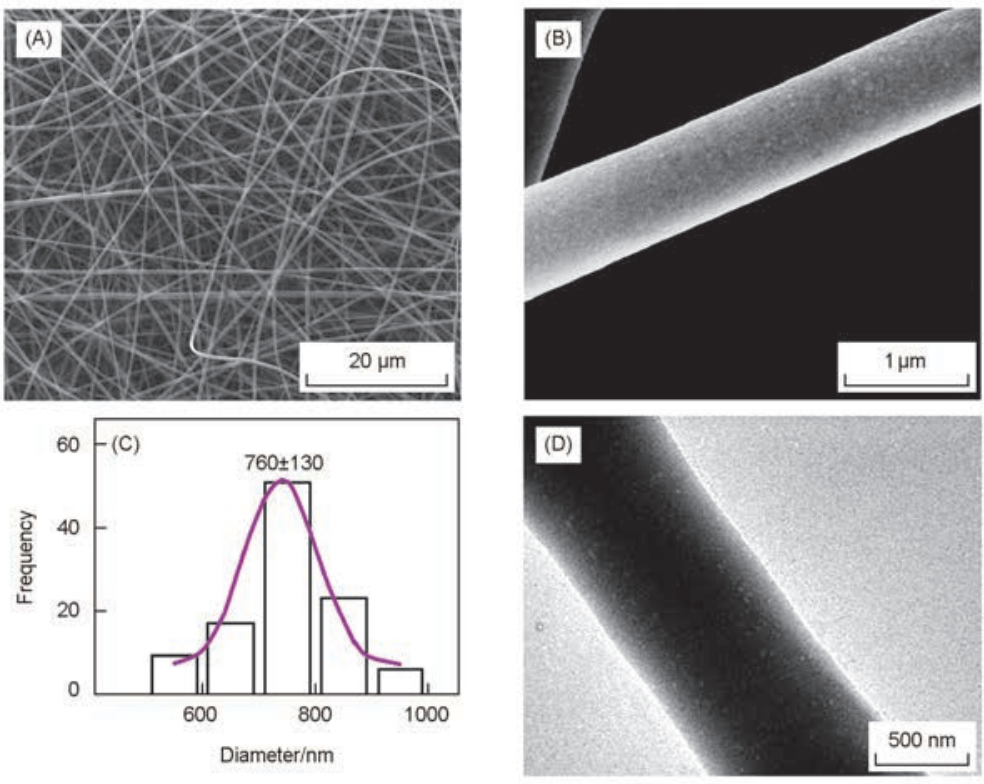

Fig.3 SEM images at a magnification of $2000(A)$ and 40000 (B), respectively, the size distribution of nanofibers' diameters(C) and TEM image of the as-prepared aspirin/Eudragit/lipid hybrid nanofibers(D)

\subsection{Physical State and Compatibility of Components in Nanofibers}

The XRD patterns of several raw materials and hybrid nanofibers are shown in Fig.4. Except for a few sharp peaks in the XRD curves of aspirin, the curves of other samples are all humps. Hence, among the three raw materials, aspirin powder was in a crystalline state. As shown in Fig.4, the aspirin powder was observed under a polarizing microscope, showing green, purple, yellow, red and other colors, which gave a hint that aspirin was a needle-like colorful crystal. Eudragit and egg yolk lecithin were in an amorphous state. Although there was the existence of phase separation in hybrid fibers, it mainly occurred between lecithin and Eudragit. The singlemolecule dispersion state of aspirin in the solution was transferred to the hybrid fibers, therefore, the existence of

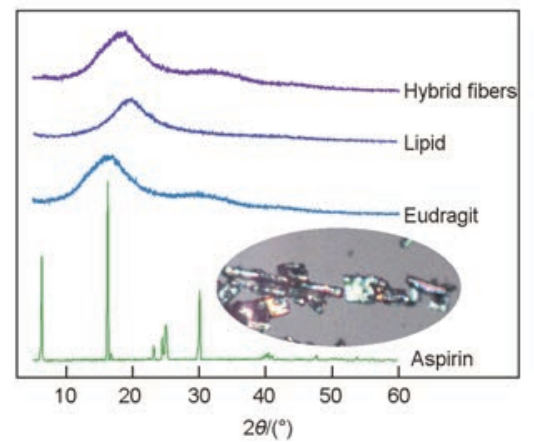

Fig.4 XRD patterns of the raw materials and their hybrid nanofibers

The inset shows the image of aspirin powders under the polarized microscope. aspirin crystals could not be detected by XRD.

The ATR-FTIR patterns of three raw materials and hybrid nanofibers as well as their molecule formula are shown in Fig.5. Benzene ring and carbonyl group exist in the aspirin molecule, so there are obvious absorption peaks at 1604, 1685 and 1755 $\mathrm{cm}^{-1}$. The main characteristic peak of Eudragit, $1727 \mathrm{~cm}^{-1}$, shows the existence of numerous carbonyl groups in the molecules. In the infrared spectra of lecithin, 2923, 1738, 1233, 1088, and $972 \mathrm{~cm}^{-1}$ corresponded to $-\mathrm{CH}_{2}$ stretching vibration, $-\mathrm{C}=\mathrm{O}$ stretching vibration, $-\mathrm{PO}_{2}$ asymmetric stretching vibration, $-\mathrm{PO}_{2}$ symmetric stretching vibration and $-\mathrm{N}\left(\mathrm{CH}_{3}\right)_{3}$ asymmetric stretching vibration absorption peaks, respectively. In hybrid nanofibers, the characteristic peaks of lecithin at 2923, 1088 and $972 \mathrm{~cm}^{-1}$, and the peak of Eudragit at $1727 \mathrm{~cm}^{-1}$ existed apparently, which illustrated that Eudragit and lecithin coexisted in the hybrid nanofibers. However, the characteristic peak of aspirin could not be
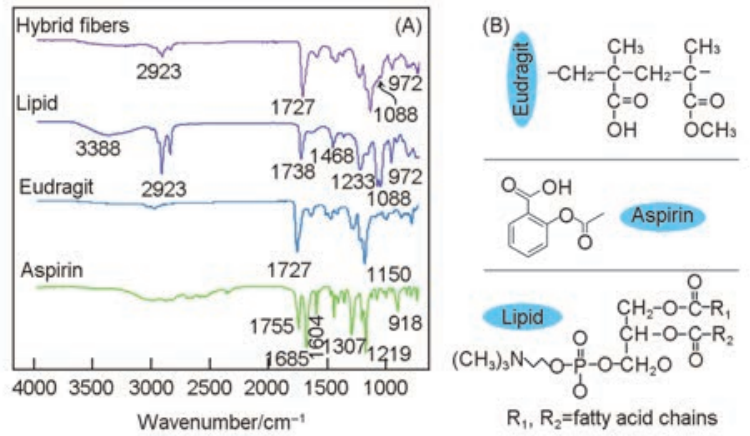

Fig.5 ATR-FTIR spectra of the three raw materials and their hybrid nanofibers $(A)$, and the molecular formula of Eudragit, aspirin, and lipid(B) 
figured out clearly, suggesting that the aspirin molecule interacted with Eudragit or lecithin through the secondary interaction(such as hydrogen bonding, hydrophobic interaction and electrostatic interaction). In other words, it was proved that aspirin had good compatibility with the two-drug carriers.

\subsection{Controlled-release Profiles of Aspirin in Nanofibers}

The in vitro controlled-release profiles of aspirin in nanofibers are shown in Fig.6(A). During the first two hours in the artificial gastric fluid environment, the aspirin released $8.7 \% \pm 3.4 \%$. The $\mathrm{pH}$-sensitive dissolution characteristics of Eudragit $^{\circledR}$ S100 can effectively prevent the drug release in stomach. As aspirin is able to easily damage gastric mucous
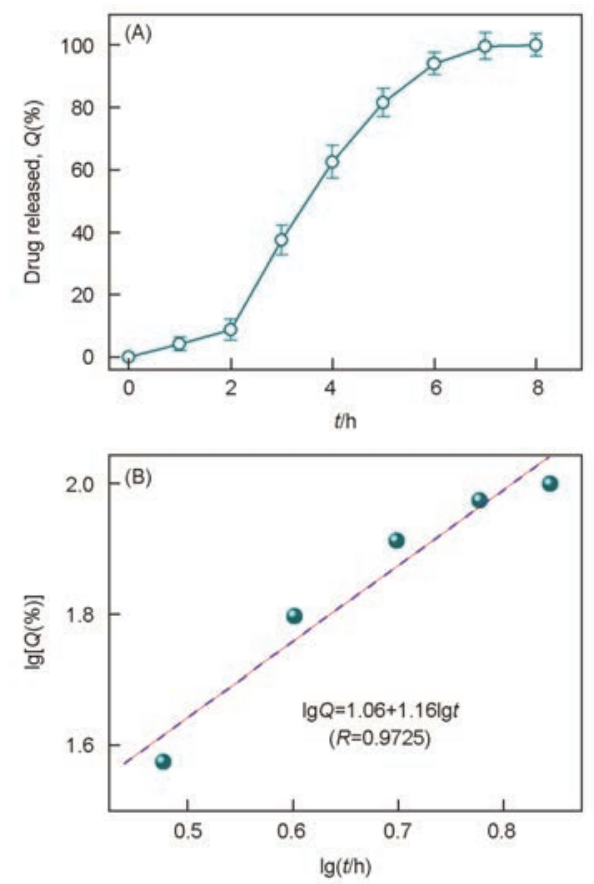

Fig.6 In vitro dissolution profiles of aspirin from the electrospun nanofibers(A) and the drug-controlled release mechanism(B) membrane, in practical applications, the delayed release of this drug can protect the gastric mucosa membrane to a certain extent. In the subsequent artificial intestinal fluid, aspirin could be released in a sustained manner. And $94.1 \% \pm 3.5 \%$ and $99.7 \% \pm 4.2 \%$ of the drug in the fibers were released at the 6 th and the 7 th hours, respectively. The drug sustainedrelease data from the 2nd hour to 6th hour were fitted using the Peppas equation ${ }^{[37]}$, the results are shown in Fig.6(B). From the equation obtained, $\lg Q=1.06+1.16 \lg t(R=0.9725)$, the controlled-release index $n$ is $1.16>0.90$, indicating that aspirin in the fiber was slowly controlled-released through dissolution mechanism. This meant that the drug was released gradually with the continuous dissolution of carrier materials.

\subsection{Energy-saving Effects and Drug Controlled- release Mechanism}

The energy-saving effects and the drug controlled-release mechanism of the prepared aspirin/Eudragit/lecithin hybrid nanofibers are shown in Fig.7. First of all, in the preparation, the structure of the coaxial spinneret with a solid core could effectively reduce energy consumption. The basic reason lied in the following aspects: (1) compared with the full-metal spinneret, the epoxy resin coated on the spinneret effectively prevented the high-voltage electrostatic energy from dispersing to the environment; (2) a Teflon rod was used to lead the fluid into the high-voltage electrostatic field, which effectively avoided the side effect of increasing energy consumption caused by the reverse capillary force of the traditional metal capillary needle spinneret, just like the reported needle-less electrospinning in the literature ${ }^{[38]}$; (3) the surface adhesion of Teflon rod was small, which could provide free surface of electrospinning fluid, meanwhile promote the small surface adhesion between the fluid and the solid rod, resulting in saving the energy consumption of high voltage electrostatic equally. Therefore, to a certain extent, the spinneret in this study combined the advantages of traditional

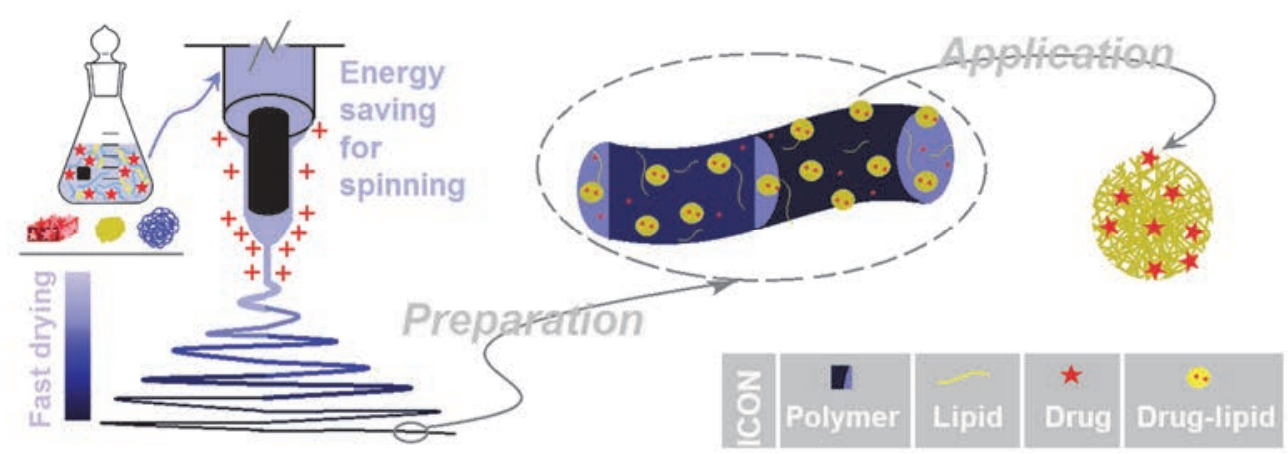

Fig.7 Energy-saving effects of the preparation of aspirin/Eudragit/lipid hybrid nanofibers and their drug-controlled release mechanism 
needle electrospinning, free surface electrospinning, and coaxial electrospinning to provide a new energy-saving and consumption-reducing process for preparing polymer nanofibers.

In essence, high voltage electrospinning is a physical process of rapid drying, during which the high-voltage electrostatic energy interacts with the fluid, promoting the solvent in the fluid to evaporate and dry rapidly, then the polymer nanofibers in a solid state are obtained. Because the drying time is extremely short, the molecular level dispersion of solutes in fluids can be effectively transferred into solid fibers to form molecular level solid dispersion. Therefore, electrospinning nanofibers are widely applied in the preparation of solid dispersions for water-insoluble drugs. However, when the compatibility of various solute molecules in a multi-solute electrospinning solution is not fine, it is possible to form a large number of discrete independent regions due to the internal phase separation during electrospinning or fiber storage. In this case, polymer-based composite should be replaced by a kind of hybrid material with multiphase coexistence.

In this study, during solvent evaporation in the bending and whipping processes at the unstable region of electrospinning, lecithin molecules are easy to agglomerate locally and spontaneously into lipid nanoparticles through electrostatic or hydrophobic interaction, along with the surrounding aspirin molecules being "involved" in, which results in the formation of lipid nanoparticles containing a large amount of drug-loaded aspirin in the Eudragit nanofibers[Fig.3(B) and (D)]. In the application of drugcontrolled release, these lipid materials are beneficial to the sustained release effect of the loaded aspirin. In the literature ${ }^{[39]}$, the drug-loaded composite materials based on Eudragit could dissolve rapidly within the suitable $\mathrm{pH}$ value, which could promote the drug to release in a pulsed manner. However, side effects of excessive blood concentration of aspirin can occur due to pulse release. In this study, the dissolved solution was mainly composed of drug-loaded lipid nanoparticles after the dissolution of Eudragit fiber due to the addition of lecithin and the formation of lipid nanoparticles in the fiber. Because of the hydrophobicity of lecithin, these nanoparticles could be further regulated to the slow release of drug molecules. Therefore, it was expected that a good colontargeted sustained-release effect could be obtained in practical application, and the possible toxic and side effects caused by high blood concentration could be avoided. Of course, this sustained-release effect does not change the controlled-release mechanism of aspirin, that is, the drug is continuously released into the bulk solution in the way of substrate corrosion, including the dissolution of Eudragit and hydrolysis of lecithin.

\section{Conclusions}

In this study, a new type of electrospinning technology was developed, which integrated the advantages of needle electrospinning, free surface electrospinning and coaxial electrospinning. The technology was characterized by a new type of coaxial spinneret with a solid core. Through the application of this spinneret, a new type of multi-component hybrid nanofibers was prepared, which was composed of drug aspirin, fiber forming polymer substrate Eudragit, and lecithin. SEM and TEM results showed that the nanofibers had good linear morphology, and there were many tiny islands due to solid phase separation. XRD results showed that the drug existed in an amorphous state in the fiber. FTIR results showed that the components had good compatibility. In vitro dissolution results verified that the drug-loaded nanofibers had dual functional effects of sustained release and colontargeted release. The prepared aspirin loaded fibers could be further converted into solid oral dosage form in the future, providing an alternative for various patients, including patients with COVID-19 infections.

\section{Acknowledgements}

This study was supported by the Natural Science Foundation of Shanghai, China(No.20ZR1439000) and the USST college student innovation projects, China(Nos.SH2020227 \& XJ2020376-2020378).

\section{Conflicts of Interest}

The authors declare no conflicts of interest.

\section{References}

[1] Song N., Wang C., Lu X. F., ACS Sustainable Chemistry \& Engineering, 2020 30, 11069

[2] Wang S. J., Luo T., Zhang X. M., Shu Y., Zhu J., Su S. P., Chemical Journal of Chinese Universities, 2017, 38(6), 990

[3] Liu J., Liu Y. B., Bukhari S. H., Ren Q., Wei C. H., Jiang X. M., Chemical Journal of Chinese Universities, 2017, 38(6), 1011

[4] Song W., Zhao B., Wang C., Lu X. F., Composites Communications, 2018, 12, 005

[5] Zhou Y., Wang X. L., Yi B. C., Yu Z. P., Yang S. Y., Shen Y. B., Zhang Y. Z., Chemical Journal of Chinese Universities, 2018, 39(7), 1554

[6] Zhao R., Lu X. F., Wang C., Composites Communications, 2018, 10, 140

[7] Qin C. P., Wang X. L., Tang H., Yi B. C., Liu C., Zhang Y. Z., Chemical Journal of Chinese Universities, 2020, 41(4), 780

[8] Yu Z. P., Yuan H. H., Yi B. C., Wang X. L., Zhangzhao W. B., Zhang Y. Z., Chemical Journal of Chinese Universities, 2018, 39(4), 807

[9] Wang Z. Q., Zhong Z. X., Ma Y. Y., Lu X. F., Wang C., Su Z. H., Chinese Journal of Polymer Science, 2020, 37, 1

[10] Wang B., Sun Z. M., Sun Q., Wang J., Du Z. X., Li C. J., Li X. Y., Environmental Pollution, 2019, 249, 851

[11] Ouyang W., Liu S., Yao K., Zhao L., Cao L., Jiang S., Hou H., Composites Communications, 2018, 9, 76

[12] Hong F., Xu H. P., Pang G., Liu G. X., Dong X. T., Yu W. S., Chemical Engineering Journal, 2020, 390, 124579

[13] Liu Z., Chen R., He J., Materials \& Design, 2016, 94, 496

[14] Zhao K., Kang S. X., Yang Y. Y., Yu D. G., Polymers, 2021, 13, 226

[15] Xu Y., Li X., Xiang H. F., Zhang Q. Q., Wang X. X., Yu M., Hao L. Y., Journal of Nanomaterials, 2020, 2, 1

[16] Jiang S., Chen Y., Duan G., Mei C., Greiner A., Agarwal S., Polymer Chemistry, 2018, 9, 2685 
[17] Kenawy E. R., Bowlin G. L., Mansfield K., Layman J., Simpson D. G., Sanders E. H., Wnek G., Journal of Controlled Release, 2002, 81, 57

[18] Vlachou M., Kikionis S., Siamidi A., Tragou K., Kapoti S., Ioannou E., Roussis V., Tsotinis A., Current Drug Delivery, 2019, 16, 79

[19] Wang K., Wen H. F., Yu D. G., Yang Y., Zhang D., Materials \& Design, 2018, 143, 248

[20] Bai Y., Wang D., Zhang Z., Pan J., Cui Z., Yu D. G., Bligh S. W. A., Polymer Testing, 2021, 93, 106872

[21] Xiao Q., Lim L. T., International Journal of Biological Macromolecules, 2018, 112,809

[22] Zheng X., Kang S., Wang K., Yang Y., Yu D. G., Wang F., Williams G. R. Bligh S. W. A., International Journal of Pharmaceutics, 2021, 596, 120203

[23] Yu D. G., Current Drug Delivery, 2021, 18, 2

[24] Wang M., Hou J., Yu D. G., Li S., Zhu J., Chen Z., Journal of Alloys and Compounds, 2020, 846, 156471

[25] Ding Y., Dou C., Chang S., Xie Z., Yu D. G., Liu Y., Shao J., Polymers, 2020 12, 2034

[26] Wang M., Li D., Li J., Li S., Chen Z., Yu D. G., Liu Z., Guo J. Z., Materials \& Design, 2020, 196, 109075

[27] Pan P., Huang Y. W., Oshima K., Yearsley M. M., Zhang J., Yu J., Arnold M., Wang L., International Journal of Molecular Science, 2018, 19, 166
[28] Haastrup P. F., Grønlykke T., Jarbøl D., Basic \& Clinical Pharmacology \& Toxicology, 2014, 116, 212

[29] Byron C., Kenneth W. M., Journal of Multidisciplinary Healthcare, 2014, 7, 137

[30] Dai Y., Ge J., Thrombosis, 2011, 2012, 1

[31] Mohamed H., Aliae A. R., Aly K. M. E., Ibrahim M. A. A., Medical Hypotheses, 2020, 144, 109975

[32] Gavillet M., Rolnik D. L., Hoffman M. K., Panchaud A., Baud D., Ultrasound in Obstetrics \& Gynecology, 2020, 55, 843

[33] Zhao Y., Jiang L., Advanced Materials, 2009, 21, 3621

[34] Aidana Y., Wang Y. B., Li J., Chang S. Y., Wang K., Yu D. G., Current Drug Delivery, 2021, 18, doi:10.2174/1567201818666210215110359

[35] Mehta P., Haj-Ahmad R., Rasekh M., Arshad M. S., Smith A., van der Merwe S. M., Li X., Chang M. W., Ahmad Z., Drug Discovery Today, 2017, 22, 157

[36] Nawzat D. A. L. J., Mohammad D. B., Jolius G., Alam A. K. M. M., Current Drug Delivery, 2019, 16, 272

[37] Peppas N. A., Narasimhan B., Journal of Controlled Release, 2014, 190, 75

[38] Liu Z., Zhao J. H., Zhou L., Xu Z. Z., Xing J., Feng Q., Recent Patents on Nanotechnology, 2019, 13, 164

[39] Chang S., Wang M., Zhang F., Liu Y., Liu X., Yu D. G., Shen H., Materials \& Design, 2020, 192, 108782 